\title{
Study of Oxidative Stress in Vitiligo
}

\author{
Anju Jain · Jyoti Mal · Vibhu Mehndiratta • \\ Ram Chander $\cdot$ Surajeet Kumar Patra
}

Received: 21 May 2009/Accepted: 30 December 2009/Published online: 14 September 2010

(C) Association of Clinical Biochemists of India 2010

\begin{abstract}
Vitiligo is an idiopathic, acquired, circumscribed, hypomelanotic skin disorder, characterized by milky white patches of different sizes and shapes. It is due to the destruction of melanocytes resulting in the absence of pigment production of the skin and mucosal surfaces. Oxidative stress has been implicated in pathophysiology of vitiligo. To study the activity of blood Superoxide dismutase (SOD) and Glutathione peroxidase (GPx) in vitiligo patients. A case-control study was conducted in which 100 patients were enrolled after written consent. 50 cases were of active vitiligo and 50 served as control (25 healthy control and 25 with stable vitiligo). SOD-In our study, among the active vitiligo cases $90 \%$ had high level of SOD and $10 \%$ had normal level of SOD. Among the stable vitiligo controls, $92 \%$ had normal level of SOD and $8 \%$ had low levels of SOD.The difference between active vitiligo cases and stable vitiligo control as well as with healthy control was statistically significant $(P$ value $<0.05)$. GPx-Among the active vitiligo cases $74 \%$ had normal GPx levels, $22 \%$ had low and only $4 \%$ had high levels of GPx. Among the stable vitiligo controls, $64 \%$ had normal GPx levels, $16 \%$ had low, and 20\% had high levels of GPx. The difference between active vitiligo cases and stable vitiligo control as well as with healthy control was statistically not significant $(P$ value $>0.05)$. Our study shows that oxidative stress is involved in the pathophysiology of
\end{abstract}

\footnotetext{
A. Jain $(\bowtie) \cdot$ S. K. Patra

Department of Biochemistry, Lady Hardinge Medical College,

New Delhi 110001, India

e-mail: dranjujain@rediffmail.com

J. Mal $\cdot$ V. Mehndiratta $\cdot$ R. Chander

Department of Dermatology, Lady Hardinge Medical College,

New Delhi 110001, India
}

vitiligo, as indicated by the high levels of serum superoxide dismutase activity.

Keywords Vitiligo - Superoxide dismutase . Glutathione peroxidase

\section{Introduction}

Vitiligo is an idiopathic, acquired, circumscribed, hypomelanotic skin disorder, characterized by milky white patches of different sizes and shapes. It is due to the destruction of melanocytes resulting in the absence of pigment production of the skin and mucosal surfaces.

The worldwide incidence of vitiligo is reported to be $0.1-2 \%$. The incidence in India is observed as $0.25-2.5 \%$ $[1,2]$. Approximately in half of the cases, the onset of disease is before the age of twenty years [3].

Clinical presentation of vitiligo include (a) segmental vitiligo, characterized by lesions that occur in a dermatomal, asymmetrical distribution (of limited clinical significance); (b) focal vitiligo, characterized by limited number of small lesions; (c) generalized vitiligo, the most common type of vitiligo where lesions occur with bilateral symmetrical distribution; and (d) universal vitiligo, almost complete depigmentation [4].

Melanocytes, the pigment producing cells are not only found in the epidermal basal layer and hair roots, but also in the uvea and retinal pigment epithelium of the eye and in the stria vascularise of inner ear. In the adult human skin, melanocytes constitute about $3-5 \%$ of epidermal cell population. Racial differences in the pigmentation intensity are not due to variations in the melanocytes density but rather due to differences in the number of melanosomes per cell and melanin content per melanosome [5]. 
The etiology of vitiligo is still not known but several theories have been proposed to explain the melanocyte destruction like genetic, neural, cytotoxic, and autoimmune theories.

Various precipitating/aggravating factors also play a role in etiopathogenesis of vitiligo like trauma (physical or emotional) or parasitic infection.

Oxidative stress is reported to play a role in progress of vitiligo but there is conflicting evidence for the same. Some researchers report increased total antioxidant levels, others report no change or even decreased levels of these markers like Superoxide Dismutase(SOD), Glutathione peroxidase (GPx), Malondialdehyde (MDA),Nitric Oxide (NO), and Catalase.

During oxidative stress, molecular oxygen $\left(\mathrm{O}_{2}\right)$ is reduced to form superoxide radicals $\left(\mathrm{O}_{2}^{*}\right)$. Further, superoxide radicals dismutase to hydrogen peroxide $\left(\mathrm{H}_{2} \mathrm{O}_{2}\right)$ either spontaneously or by the action of Superoxide Dismutase (SOD).

In epidermal cells, $\mathrm{H}_{2} \mathrm{O}_{2}$ levels increase in response to oxidative stress from environmental trauma, such as ultraviolet radiation B (290-320 mm) [6].

Moreover, there is basal level of oxidants in cells that is by-product of normal endogenous metabolic processes. Although a system of enzymatic and non enzymatic antioxidants provides protection against reactive oxygen species (ROS), an imbalance between oxidants and antioxidants can lead to disruption of cellular function [7].

Our study aims to study the clinical profile and the role of oxidative stress in active and stable cases of vitiligo and in healthy controls.

\section{Materials and Methods}

This prospective case-control study was conducted jointly in the Department of Dermatology, Venerology, and Leprosy and Department of Biochemistry, Lady Hardinge Medical College and Smt Sucheta Kriplani Hospital, New Delhi, India after institutional ethical clearance.

One hundred subjects were enrolled in our study with an informed consent. They were divided into two groups.

Group 1: 50 clinically diagnosed cases of vitiligo (active vitiligo) of all types based upon the inclusion criteria

Group 2: 50 controls, out of these, 25 were Vitiligo cases with stable disease (stable Vitiligo) and remaining 25 were healthy controls.

The inclusion criteria were

- All clinically diagnosed cases of vitiligo of all types.

- Patients who have active disease at the time of study i.e., duration of the disease is less than 6 months and/or the appearance of new lesions and/or increase in the size of the existing lesions in the last 6 months.

- All patients $>15$ years of age of either sex.

- Untreated patients or those patients who have not received any kind of systemic topical treatment in the last 3 months for vitiligo.

The exclusion criteria were

- Patients who have stable vitiligo i.e., No progression of existing disease or appearance of new lesions in the last six months.

- Patients who have been on topical or systemic treatment during the last 3 months for vitiligo.

- Patients who have any concomitant systemic or dermatological disease.

The control group consists of

Group 1: All healthy volunteers

Group 2: Stable Vitiligo cases

Detailed history was taken and clinical examination was done in all subjects of the study group. Venous blood samples were collected for estimation of SOD and GPx.

Estimation of Superoxide Dismutase (SOD)

Assay of superoxide dismutase (SOD) in whole blood samples was done according to the method of Williams J.A et al. [8] 1983. Randox Kit.The assay was done according to the instructions provided by the manufacturer.

This method employs xanthine and xanthine oxidase (XOD) to generate superoxide radicals which react with 2-(4-iodophenyl)-3-(4-nitrophenol)-5-phenyltetrazolium chloride (INT) to form a red formazan dye. The superoxide dismutase activity is then measured by the degree of inhibition of this reaction. One unit of SOD is that which causes a $50 \%$ inhibition of the rate of the reduction of INT under the conditions of the assay.

\section{Estimation of Glutathione Peroxidase (GPx)}

Assay of Glutathione Peroxidase (GPx) in whole blood samples was done by the Paglia and Valentine method [9] using Randox kit. The assay was done according to the instruction provided by the manufacturer.

Glutathione Peroxidase (GPx) catalyses the oxidation of Glutathione (GSH) by Cumene Hydroperoxide. In the presence of Glutathione Reductase (GR) and NADPH the oxidised Glutathione (GSSG) is immediately converted to the reduced form with a concomitant oxidation of NADPH to NADP + . The decrease in the absorbance at $340 \mathrm{~nm}$ is measured in spectrophotometer. 
Table 1 SOD and GPx in study groups

\begin{tabular}{|c|c|c|c|c|c|c|}
\hline \multirow[t]{2}{*}{ Study group } & \multicolumn{3}{|l|}{ SOD } & \multicolumn{3}{|l|}{ GPx } \\
\hline & $\begin{array}{l}\text { Low }<164 \\
\mathrm{U} / \mathrm{ml}(\%)\end{array}$ & $\begin{array}{l}\text { Normal 164-240 } \\
\text { U/ml(\%) }\end{array}$ & $\begin{array}{l}\text { High }>240 \\
\mathrm{U} / \mathrm{ml}(\%)\end{array}$ & $\begin{array}{l}\text { Low }<4171 \\
\mathrm{U} / 1(\%)\end{array}$ & $\begin{array}{l}\text { Normal 4171-10881 } \\
\text { U/l(\%) }\end{array}$ & $\begin{array}{l}\text { High }>10881 \\
\text { U/1 }\end{array}$ \\
\hline Vitiligo active cases $(n=50)$ & 0 & $5(10)$ & $45(90)$ & $25(50)$ & $25(50)$ & 0 \\
\hline Vitiligo stable control $(n=25)$ & $2(8)$ & $23(92)$ & 0 & $13(52)$ & $12(48)$ & 0 \\
\hline Healthy control $(n=25)$ & $3(12)$ & $16(64)$ & $6(24)$ & $14(56)$ & $11(44)$ & 0 \\
\hline
\end{tabular}

Bold value indicates statistically significant

\section{Statistical analysis}

The statistical analysis was performed using SPSS version 12 software. The data are expressed as mean $\pm \mathrm{SD}$. The Chi square values and $\mathrm{p}$ values are used for the interpretation of results. $P$ value $<0.05$ is considered as significant.

\section{Results}

In our study, a female preponderance was seen in patients of Vitiligo (68\% female and $32 \%$ male).

The maximum number of vitiligo cases (54\%) occurs in the age group of 16-25 years followed by $16 \%$ in 26-35 years. The age of onset of vitiligo was maximum in 11-20 years.

Among the vitiligo cases, disease onset was seen in age group of 11-20 years (44\%) followed by $24 \%$ in 21-30 years. The incidence is lower in higher age group.

The most common type of Vitiligo seen clinically is Vitiligo vulgaris (52\%) followed by $34 \%$ Vitiligo acrofacialis. Among the stable vitiligo control showed $36 \%$ vulgaris, $24 \%$ acrofacialis, and $20 \%$ focal vitiligo.

Premature graying of hairs was significantly associated with vitiligo. Vitiligo active or stable showed no significant association with Rheumatic factor or antinuclear antibody or Thyroid Stimulating Hormone (TSH) levels.

Blood SOD and GPx normal ranges as reported by the manufacturers are 164-240 U/ml and 4171-10881 U/l, respectively.

In our study, the mean values (Mean $\pm \mathrm{SD}$ ) of SOD $(\mathrm{U} / \mathrm{ml})$ are $367 \pm 25$ in active Vitiligo, $300 \pm 83$ in Vitiligo control and $237 \pm 45$ in healthy control .

The values (Mean $\pm \mathrm{SD}$ ) of GPx (U/l) in various groups are $4011 \pm 498,3990 \pm 459$ and $3945 \pm 552$ in active Vitiligo cases, Vitiligo control and healthy control respectively.

Table 1 shows the values of SOD and GPx in low, normal and high ranges in the various groups.

The comparative analysis by chi square test among the groups shows SOD to be significant $(P$ value $<0.05)$ in
Table 2 Comparative analysis between study groups

\begin{tabular}{lll}
\hline Study groups & \multicolumn{2}{l}{$P$ value } \\
\cline { 2 - 3 } & SOD & GPx \\
\hline Vitiligo active cases and healthy controls & $<0.05$ & $>0.05$ \\
Vitiligo active cases and vitiligo stable controls & $<0.05$ & $>0.05$ \\
\hline
\end{tabular}

$P$ value $<0.05$ significant

active Vitiligo cases when compared with Vitiligo controls or healthy controls. Table 2

\section{Discussion}

In our study, we observed that the most common age group affected by active Vitiligo is 16-25 years and it is more prevalent in females. This is in accordance with the findings of Shajil [10] and Daneshpazhooh [11] who also noted female preponderance. Among the Vitiligo cases in majority of patients, the onset of disease was between 11-20 years of age. This is supported by Gopal and Rao et al. [12]. Early onset of the disease may be due to genetic predisposition. Vitiligo vulgaris was found to be the most common clinical type of Vitiligo as has also been reported by Beazley et al. [13] and Shajil et al. [10].

We observed significantly high levels of superoxide dismutase (SOD) in active Vitiligo cases as compared to stable Vitiligo and healthy controls. Hazneci et al. [14] also reported SOD activity was significantly higher in active Vitiligo patient as compared to healthy controls. This suggests that there is an imbalance in the oxidant-antioxidant system (oxidative stress) due to disease activity resulting in high level of blood superoxide dismutase level. However, Glutathione peroxidase did not show any significant association in the active cases and stable cases of Vitiligo. Previous studies reveal an inconsistent pattern in the level of GPx. Passi et al. [15] reported an increased level of GPx whereas Agrawal et al. [16] reported a decreased level in Vitiligo cases.

However, the exact role of oxidative stress and role of antioxidant as therapeutic agents in vitiligo is possible only after large multicentric studies. 


\section{References}

1. Agrawal D, Sahani MH, Gupta S, Begum R. Vitiligo etipathogenesis and therapy: a review. J Maharaja Sayajirao Univ of Baroda. 2001;48:97-106.

2. Moscher DB, Fitzpatrick TB, Ortonne JP, Hori Y. Hypomelnosis and hypermelanosis. In: Eisen AZ, Wolff K, Austen KF, Goldsmith LA, Kats SI, Fitzpatrick TB, editors. Dermatology in general medicine. New York: MC Graw Hill; 1999. p. 945-1017.

3. Lerner AB. Vitiligo. J Invest Dermatol. 1959;32(2):285-310.

4. Mosher DB, Fitzpatrick TB, Hori Y and Ortonne JP. Disorders of melanocyte. Dermatology in General medicine, $4^{\text {th }}$ Ed. New York: McGraw Hill,1993, 903-995.

5. Jimbow K, Quevedo WC, Fitzpatrick TB and Szabo G. Biology of melanocytes. Dermatology in General medicine, $4^{\text {th }}$ Ed. New York: McGraw Hill; 1993: 261-289.

6. Peus D, Vasa RA, Meves A, Pott M, Beyerle A. $\mathrm{H}_{2} \mathrm{O}_{2}$ is an important mediator of UVB induced by EGF receptor phosphorylation in cultured melanocytes causing damage to biomolecules. J Invest Dermatol. 1998;110:966-71.

7. Lopez TM, Thiele JJ, Shindo Y, Han D, Packer L. Topical application of alpha-tocopherol modulates the antioxidant network diminishes ultraviolet-induced oxidative damage in murine skin. Br J Dermatol. 1998;138:207-15.

8. Wooliams JA, Wiener G, Anderson PH, Mc Murray CH. Variation in the activities of glutathione peroxidase and superoxide dismutase and in the concentration of copper in the blood in various breed crosses of sheep. Res Vet Sci. 1983;34:253-6.

9. Paglia DE, Valentine WN. Studies on the quantitative and qualitative characterization of erythrocyte glutathione peroxidase. J Lab Clin Med. 1967;70:158.

10. Shajil EM, Agrawal D, Vagadia K, Marfatia YS, Begum R. Vitiligo: clinical profiles in vadodara. Indian J Dermatol Venerol. 2006;51(2):100-4.

11. Daneshpazhooh M, Mostofizadeh GM, Behjati J, Akhyani M, Robati RM. Antithyroid peroxidase antibody and Vitiligo: a controlled study. BMC dermatology. 2006;6:3.

12. Gopal KVT, Rao GRR, Kumar HK, Rao MVA, Vasudev P. Vitiligo: A part of systemic autoimmune process. Indian J Dermatol Venerol. 2007;73:162-5.

13. Beazley WD, Gaze D, Panske A. Serum Selenium levels and blood glutathione peroxidase activities in vitiligo. British $\mathbf{J}$ of Dermatol. 1999;141:301-3.

14. Hazneci E, Karabulut AB, Öztürk C, Batçioğlu K, Doğan G, Karaca S, Eşrefoğlu M. A comparative study of superoxide dismutase, catalase, and glutathione peroxide activities and nitrate levels in vitiligo patients. Int J Dermatol. 2005;44(8):636-40.

15. Passi S, Grandinetti M, Maggio F, Stancato A, Luca C. Epidermal oxidative stress in vitiligo. Pigment Cell Res. 1998;11(2):81-5.

16. Agrawal D, Shajil EM, Marfatia YS, Begum R. Study on the antioxidant status of vitiligo patients of different age groups in Baroda. Pigment Cell Res. 2004;17(3):289-94. 